\title{
Heavy Ion Beam Probing - the diagnostics for internal measurements of the electric potential and turbulence in toroidal plasma devices
}

\author{
A.V. Melnikov ${ }^{1}$ \\ National Research Centre "Kurchatov Institute" \\ 123182, Moscow, Russia \\ National Research Nuclear University MEPhI \\ 115409, Moscow, Russia \\ E-mail: meInikov 070yahoo.com

\section{L.G. Eliseev} \\ National Research Centre "Kurchatov Institute" \\ 123182, Moscow, Russia \\ E-mail: reonideyahoo.com
}

\section{S.E. Lysenko}

National Research Centre "Kurchatov Institute" 123182, Moscow, Russia

E-mail: Iysenko_SE@nrcki.ru

\section{S.V. Perfilov}

National Research Centre "Kurchatov Institute" 123182, Moscow, Russia

E-mail: stas_8930pochtamt.ru

\section{V.N. Zenin}

National Research Centre "Kurchatov Institute" 123182, Moscow, Russia

E-mail: vitalyzenin@mail.ru

\section{L.I. Krupnik}

Institute of Plasma Physics, KIPT

61108, Kharkov, Ukraine

E-mail: krupnikeipp.kharkov.ua

\section{A.A. Chmyga}

Institute of Plasma Physics, KIPT

61108, Kharkov, Ukraine

E-mail: chmygalipp.kharkov.ua

\section{A.D. Komarov}

Institute of Plasma Physics, KIPT

61108, Kharkov, Ukraine

${ }^{1}$ Speaker 
E-mail: hibpeipp.kharkov.ua

\section{A.S. Kozachek}

Institute of Plasma Physics, KIPT

61108, Kharkov, Ukraine

E-mail: hibpeipp. kharkov.ua

\section{S.M. Khrebtov}

Institute of Plasma Physics, KIPT

61108, Kharkov, Ukraine

E-mail: hibpeipp. kharkov.ua

\section{Yu.I. Tashchev}

Institute of Plasma Physics, KIPT

61108, Kharkov, Ukraine

E-mail: hibpeipp. kharkov.ua

\section{A.I. Zhezhera}

Institute of Plasma Physics, KIPT

61108, Kharkov, Ukraine

E-mail: hibpeipp.kharkov.ua

\section{J.M. Barcala}

Technology Department, CIEMAT

28040, Madrid, Spain

E-mail: miguel.barcala@ciemat.es

C. Hidalgo

Fusion National Laboratory, CIEMAT

28040, Madrid, Spain

E-mail: carlos.hidalgo@ciemat.es

\section{J. Lopez}

Fusion National Laboratory, CIEMAT

28040, Madrid, Spain

E-mail: j. Iopez@ciemat.es

\section{A. Molinero}

Technology Department, CIEMAT

28040, Madrid, Spain

E-mail: antonio.molinero@ciemat.es

\section{J.L. de Pablos}

Fusion National Laboratory, CIEMAT

28040, Madrid, Spain

E-mail: jl.dePablos@ciemat.es 
Heavy Ion Beam Probing (HIBP) is a unique diagnostics for core plasma potential. Advanced HIBP is operating now in the T-10 tokamak ( $a=0.3 \mathrm{~m}, R=1.5 \mathrm{~m}, B_{\mathrm{t}}=1.5-2.4 \mathrm{~T}, I_{\mathrm{p}}=140-330 \mathrm{kA}$, $\left.P_{\mathrm{ECRH}}<1.2 \mathrm{MW}\right)$ and TJ-II flexible heliac $\left(<a>=0.22 \mathrm{~m},<R>=1.5 \mathrm{~m}, B_{\mathrm{t}}=1 \mathrm{~T}, P_{\mathrm{ECRH}} \leq 0.6 \mathrm{MW}\right.$, $\left.P_{\mathrm{NBI}} \leq 1 \mathrm{MW}\right)$.

The fine focused $(<1 \mathrm{~cm})$ and intense $(100 \mu \mathrm{A})$ beams provide the measurements in the wide density interval $n_{\mathrm{e}}=(0.3-5) \times 10^{19} \mathrm{~m}^{-3}$, while the advanced control system for primary and secondary beams provides the measurements in the wide range of the plasma current in T-10 and magnetic configurations in TJ-II, including Ohmic, ECR and NBI heated plasmas.

The time evolution of the radial profiles and/or local values of plasma parameters from High Field Side (HFS) to Low Field Side (LFS), $-1<\rho<1$, is observed in TJ-II by $125 \mathrm{keV} \mathrm{Cs}^{+}$ions in a single shot, while LFS $(+0.2<\rho<1)$ profiles are observed in $\mathrm{T}-10$ by $300 \mathrm{keV} \mathrm{Tl}{ }^{+}$ions.

Multi-slit energy analyzers provide simultaneously the data on plasma potential $\varphi$ (by beam extra energy), plasma density $n_{\mathrm{e}}$ (by beam current) and $B_{\text {pol }}$ (by beam toroidal shift) in poloidally shifted sample volumes. Thus $E_{\mathrm{pol}}=\left(\varphi_{1}-\varphi_{2}\right) / x, x \sim 1 \mathrm{~cm}$, and the electrostatic turbulent particle flux $\Gamma_{E \times B}(t)=1 / B_{t} \cdot \tilde{n}_{e}(t) \tilde{E}_{\mathrm{pol}}(t)$ is derived. The density oscillations cross-phase produces the phase velocity of poloidal propagation of perturbation or plasma rotation and the poloidal mode number $m$.

Dual HIBP, consisting of two identical HIBPs located $1 / 4$ torus apart, recently installed in TJ-II, provides the long-range correlations in core plasma parameters.

Low noise high gain $\left(10^{7} \mathrm{~V} / \mathrm{A}\right)$ preamplifiers with $300 \mathrm{kHz}$ bandwidth and $2 \mathrm{MHz}$ sampling allows us to study broadband turbulence and quasi-coherent modes like Geodesic Acoustic Modes, Alfven Eigenmodes, Suprathermal electron induced modes, etc.

Finally, HIBP becomes multipurpose diagnostics to study plasma electric potential and turbulence properties in the middle size toroidal devices.

First EPS Conference on Plasma Diagnostics - $1^{\text {st }}$ ECPD

14-17 April 2015,

Villa Mondragone, Frascati (Rome) Italy 


\section{Introduction}

HIBP $[1,2]$ is a unique tool for the direct measurement of the plasma potential in the core plasma of fusion devices. HIBP was first introduced in 1970s [3], it was later developed to the multichannel version to study the turbulent particle flux [4]. This report presents the further HIBP development and application to the T-10 tokamak [5] ( $a=0.3 \mathrm{~m}, R=1.5 \mathrm{~m}, B_{\mathrm{t}}=1.5-2.4 \mathrm{~T}, I_{\mathrm{p}}=140-$ $\left.330 \mathrm{kA}, P_{\mathrm{ECRH}}<1.2 \mathrm{MW}\right)$ and TJ-II stellarator [6] $\left(<a>=0.22 \mathrm{~m},<R>=1.5 \mathrm{~m}, B_{\mathrm{t}}=1 \mathrm{~T}, P_{\mathrm{ECRH}} \leq 0.6\right.$ $\left.\mathrm{MW}, P_{\mathrm{NBI}} \leq 1 \mathrm{MW}\right)$. It discusses the diagnostics capabilities and limitations and also presents the examples of recent measurements of plasma potential and turbulence characteristics.

\section{Plasma potential profiles}

HIBP is capable to measure potential time evolution in the fixed sample volume (SV) and radial profiles as well. The spatial resolution is about $1 \mathrm{~cm}$, time resolution is $0.5-1 \mu \mathrm{s}$. HIBP schematics is shown in figure 1 by example of the TJ-II flexible heliac.

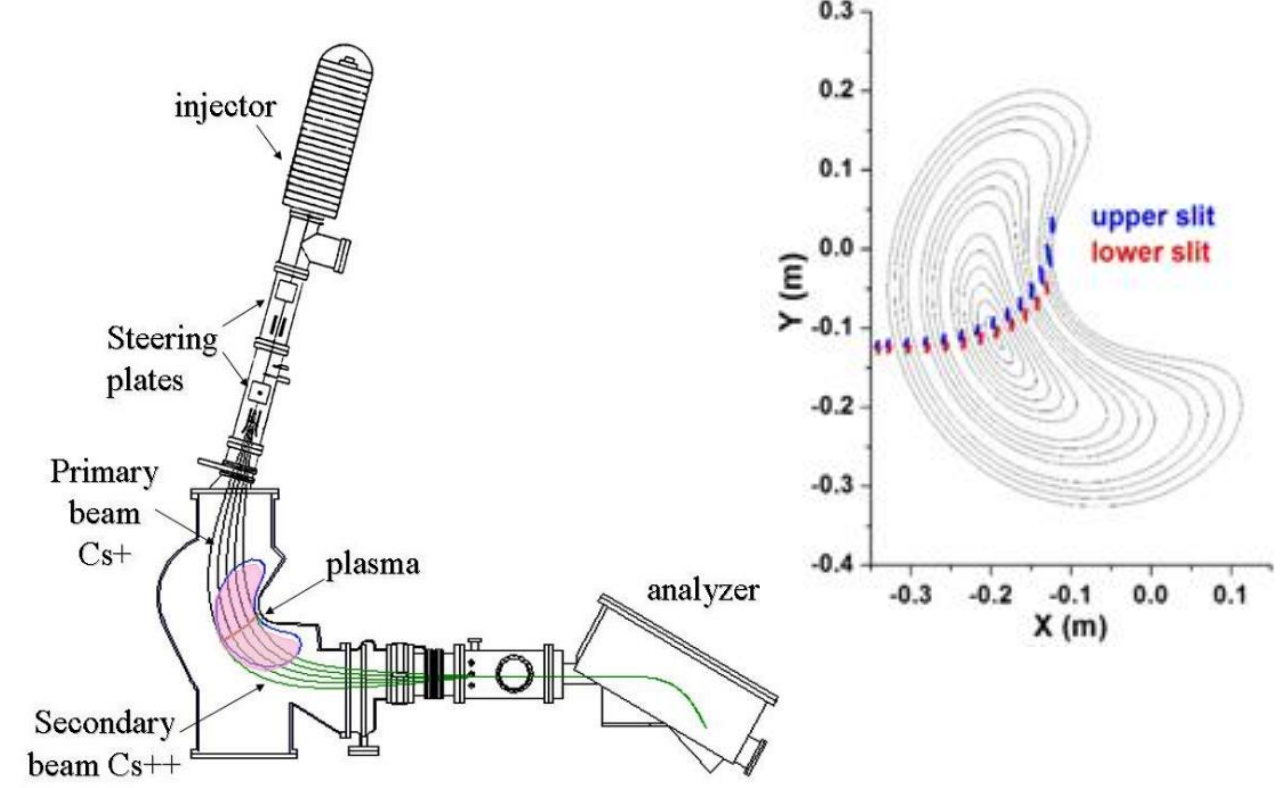

Figure 1. HIBP experimental set-up at TJ-II. Black curves are trajectories of primary $\left(\mathrm{Cs}^{+}\right)$ions, green ones - of secondary $\left(\mathrm{Cs}^{++}\right.$ions. Inset shows detector lines for the poloidally resolved potential and density measurements.

The time evolution of the radial profiles from Low Field Side (LFS) to the High Field Side $(-1<\rho<1)$ is observed in TJ-II with $125 \mathrm{keV} \mathrm{Cs}^{+}$ions in a single shot. The typical scanning time is $10 \mathrm{~ms}$. In T-10, 4-6 repetitive pulses produce profile fragments with various beam $\mathrm{Tl}^{+}$energies from 80 to $300 \mathrm{keV}$, which unify to the radial profiles in LFS $(+0.2<\rho<1)$, as shown in figure 2 .

The fine focused $(<1 \mathrm{~cm})$ and intense $(100 \mu \mathrm{A})$ beams provide the measurements in the wide density interval $n_{\mathrm{e}}=(0.3-5) \times 10^{19} \mathrm{~m}^{-3}$, while the advanced control system for primary and secondary beams provides the measurements in the wide range of the plasma current in T-10 and magnetic configurations in TJ-II, including Ohmic, ECR and NBI heated plasmas [7-9]. 

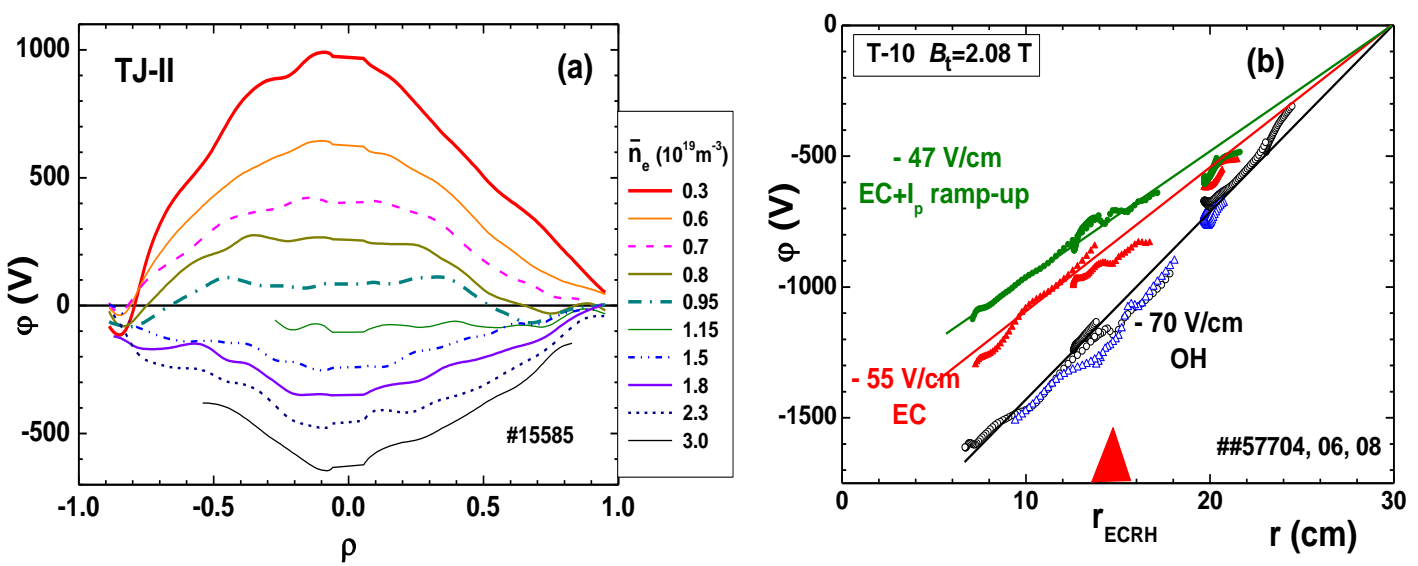

Figure 2. Evolution of the electric potential profiles in the plasmas with ECR and NBI heating on TJ-II (a) and with Ohmic and ECR heating in T-10 (b).

\section{Plasma oscillations}

Beam detector in the multi-slit electrostatic energy analyser is designed to measure simultaneously the data on plasma potential $\varphi$ (by beam extra energy), plasma density $n_{\mathrm{e}}$ (by beam current) and $B_{\mathrm{pol}}$ (by beam toroidal shift) in poloidally shifted sample volumes. There are from 2 to 5 slits (spatial channels) in operation. The two slits measurement scheme is shown in figure 1.

Low noise high gain $\left(10^{7}\right.$ V/A) preamplifiers with $300 \mathrm{kHz}$ bandwidth and $2 \mathrm{MHz}$ sampling allows us to study broadband turbulence and quasi-coherent modes like Geodesic Acoustic Modes (GAM), Alfvén Eigenmodes (AE), Suprathermal electron induced modes, etc.

\subsection{Quasi-coherent modes: GAMs and AEs}

HIBP is sensitive to the local plasma potential and density perturbation associated with GAM. GAM amplitude in plasma potential depends on the electron density and vary from the noise level ( $<10$ V) up to $\sim 100$ V. Figure 3 (left) shows the Fourier Power Spectral Density (PSD) for the potential and density oscillations with a frequency resolution $<2 \mathrm{kHz}$. GAM is observed as a pronounced frequency peak in potential with a high contrast respect to the broadband turbulence, while the MHD mode is mainly seen in plasma density. The high time resolution (1-2 $\mu \mathrm{s})$ allows us to resolve the GAM intermittent structure with a typical length of the wave packages $\sim 1-2 \mathrm{~ms}$.

HIBP shows that GAMs on T-10 have a radially uniform spatial structure of potential perturbation and the $\pi / 2$ phase shift between the plasma potential and density.

Due to its capability to measure simultaneously potential, $B_{\mathrm{pol}}$ and density oscillations, HIBP is the most direct diagnostics to study Alfvén Eigenmodes, an electromagnetic waves, propagating along the magnetic field lines of plasma configuration and producing the oscillatory components of $E_{\mathrm{r}}, B_{\mathrm{pol}}$ and plasma pressure (density). AEs are visible in all three HIBP 

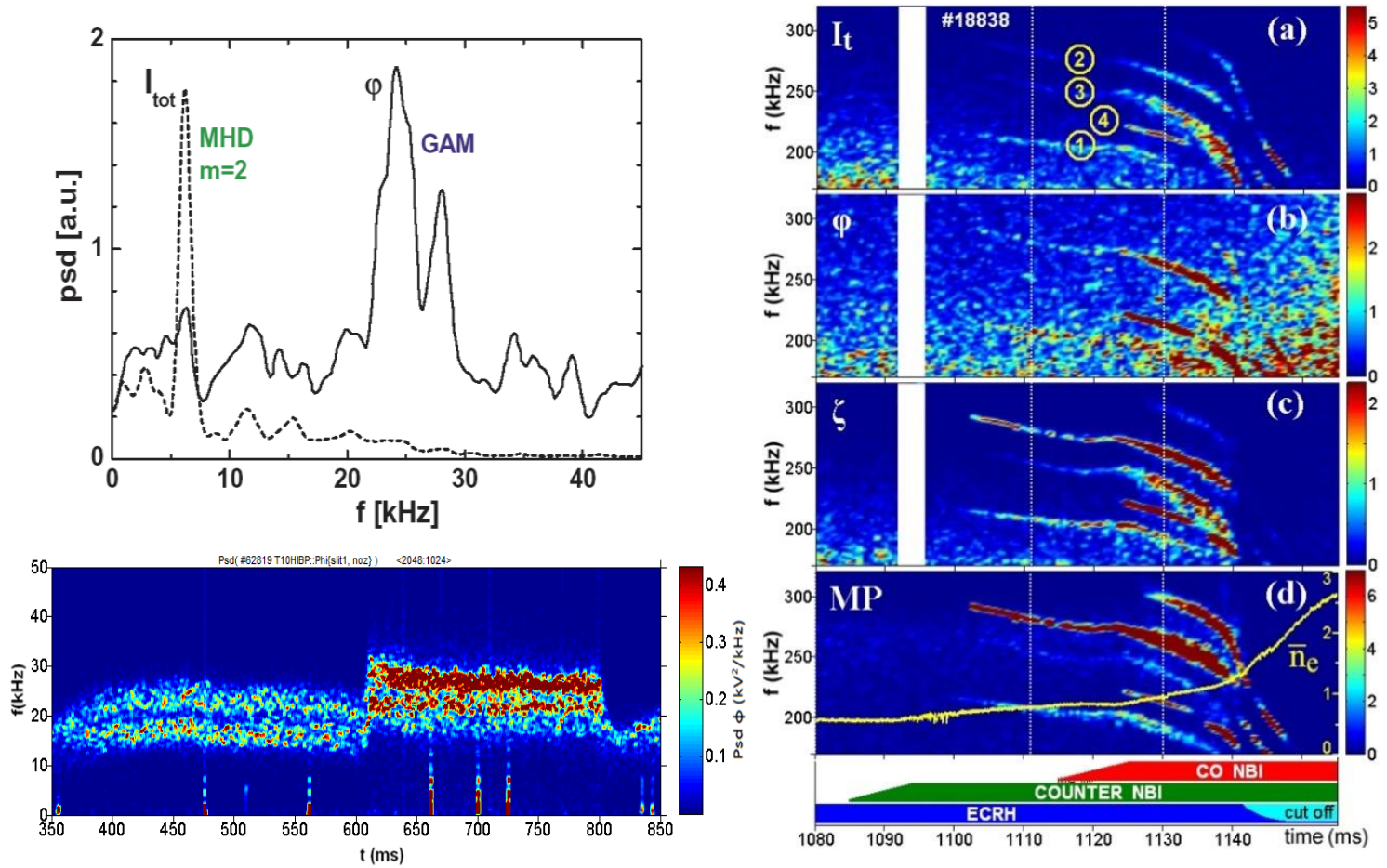

Figure 3. Left: T-10 data. Left up. Potential and density power spectra for GAM. Left down potential spectrogram shows GAM evolution in the pulse with ECRH. The GAM intermittent structure is observed. Right: TJ-II data. PSD spectrograms of HIBP $(\rho=-0.5)$ and Magnetic Probe (MP) signals in arb. un. Alfvén Eigenmodes are pronounced: (a) on the total secondary beam current $I_{t}$ proportional to $n_{e}$; (b) on the potential $\varphi$; (c) on the toroidal shift of secondary beam $\zeta$ proportional to $B_{\text {pol; }}$ (d) on the MP signal.

parameters as presented in their spectrograms in figure 3 (right). Various modes, marked with numbers are clearly detectable in the NBI heated phase of the discharge. The spatial scan of the sample volume allows us to find a radial location for each mode.

\subsection{Turbulent particle flux}

Multi-slits energy analyzer provides the oscillatory component of the plasma potential $\varphi$, and density $\tilde{n}_{e}(t)$ in poloidally shifted sample volumes, see figure 1 . Thus $E_{\mathrm{pol}}=\left(\varphi_{1}-\varphi_{2}\right) / x, x \sim 1$ $\mathrm{cm}$, and the electrostatic turbulent particle flux $\Gamma_{E \times B}(t)=1 / B_{t} \cdot \tilde{n}_{e}(t) \tilde{E}_{\mathrm{pol}}(t)$ is derived. Frequency resolved turbulent particle flux, which is taken as [10]: $\Gamma_{E \times B}(f)=-2 / B_{t o r} \cdot \operatorname{Re}\left(S_{n E}\right)$, where $S_{n E}$ is the Fourier cross-spectrum of density and $E_{\text {pol }}$, allows us to get a contribution of the broadband turbulence [11] and that of the quasicoherent modes [12]. Figure 4 (a) shows the frequency resolved flux for the NBI discharge of TJ-II. It shows that the broadband turbulence at $f_{B B}<250$ $\mathrm{kHz}$ mainly produce the outward flux, denoted by red color. On top of that, the AEs are clearly visible in the spectrogram as a set of quasi-monochromatic modes. Figure 4 (a) shows that the contribution of the specific modes may vary: there are outward, inward and zero flux presented for different AEs. The direction and the value of the flux contribution for specific AEs is it's specific characteristics. It's value depends on the cross-phase $\theta_{\text {ne Epol }}$ between $n_{\mathrm{e}}$ and $E_{\text {pol }}$ oscillations, as seen in the figures 4 (b, c, d). 

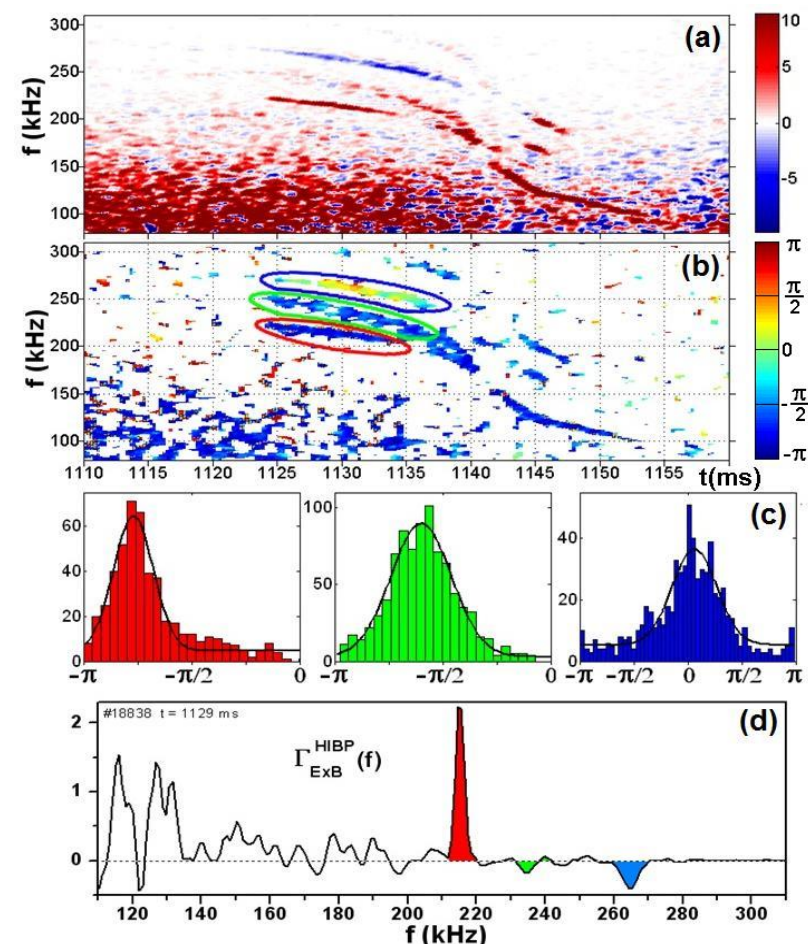

(d)
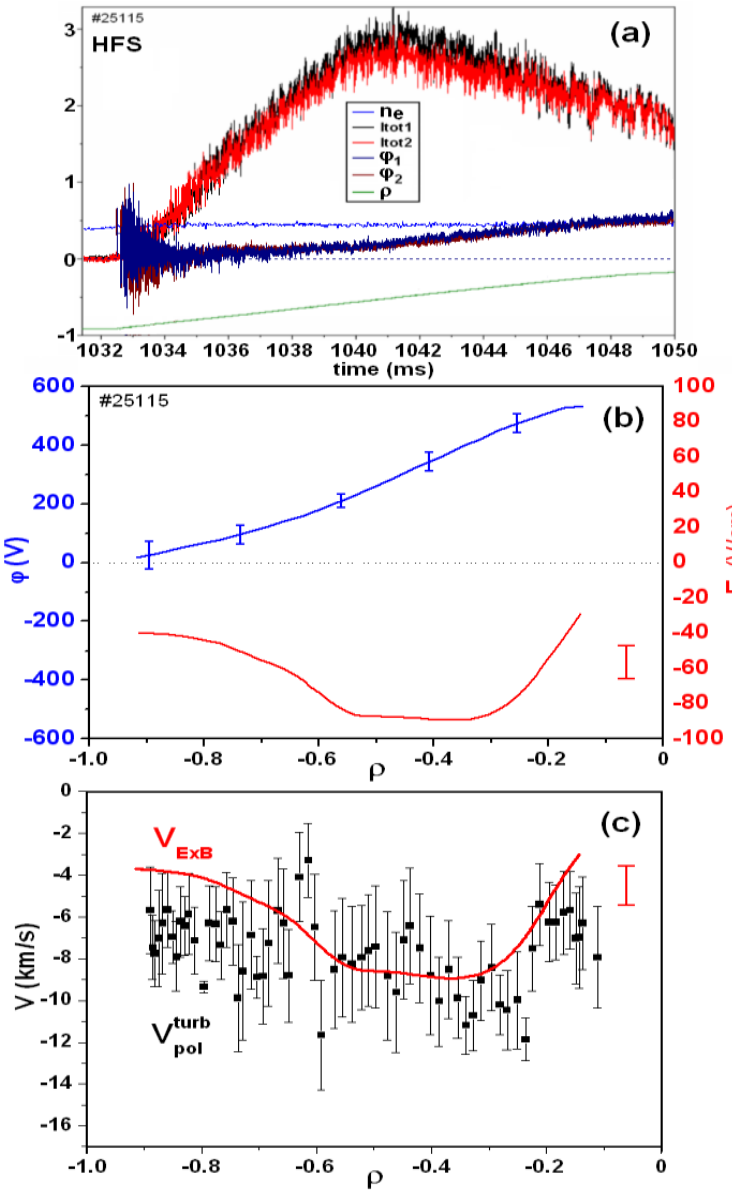

Figure 4. a) Frequency resolved turbulent particle flux (in arb. units) in the NBI sustained dischargeof TJ-II. Red colour means outward flux, blue - inward flux.

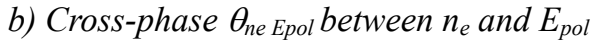
oscillations. Only the points with high $\mathrm{Coh}_{n e \text { Epol }}>0.3$ are shown. The colour bar is in radians. Three chosen AEs are marked by colour ovals.

c) The histograms of the cross-phase for each marked AEs with corresponding colours, indicating flux direction.

d) PSD of the turbulent particle flux, taken at some time instant, averaged over $1 \mathrm{~ms}$.

Figure 5. Comparison of $V_{\text {phase }}^{\text {turb }}$ and $V_{E \times B}$ measured by HIBP in TJ-II.

(a) raw signals of HIBP currents (red and black) and potentials (blue and brown) obtained during radial scan. The density time trace is shown as thin blue curvs, the SV radial position is in green;

(b) smoothed potential (blue) and $E_{r}$ (red) radial profiles;

(c) $V_{p o l}$ and $V_{E \times B}$ radial profiles. Both of $V_{p o l}$ and $V_{E \times B}$ are directed to ion diamagnetic drift. 


\subsection{Poloidal phase rotation for the broadband turbulence}

The density oscillations cross-phase $\theta_{1,2}(f)=\arg \left\{P_{1,2}(f)\right\}$, where $P_{1,2}(f)=n_{1}^{*}(f) n_{2}(f)$ produces poloidal propagation velocity of perturbation or plasma rotation: $V_{\text {phase }}^{\text {turb }}=\Delta x \cdot 2 \pi f / \theta_{1,2}$ [13]. Here we consider $\tilde{n}_{e} / n_{e} \approx \tilde{I}_{\text {tot }} / I_{\text {tot }}$, where $\tilde{I}_{t o t} / I_{\text {tot }}$ is the relative oscillations of the beam current. Figure 5 shows the radial distribution of the plasma turbulence rotation for $0<f<150 \mathrm{kHz}$ in the TJ-II stellarator. Plasma potential profile is measured altogether with the density turbulence, see figure 5 (b). Figure 5 (c) shows that $V_{\text {phase }}^{\text {turb }}$ and $V_{E \times B}$ are in coincidence with an experimental accuracy over the whole radial interval.

\section{Conclusions}

- HIBP operates in the T-10 tokamak and TJ-II stellarator with high spatial and temporal resolution over the whole radial range in wide operational domain of the machines.

- It provides the time evolution of the full radial mean profiles of plasma potential (or $E_{r}$ ) in LFS on T-10 and in both LFS and HFS in TJ-II.

- The new multislit energy analyzers simultaneously measure the oscillatory component of three parameters: plasma potential, density and $B_{\text {pol }}$.

- Thus the poloidal mode number and rotation velocity of the density broadband turbulence and quasicoherent modes are retrieved.

- The frequency resolved turbulent particle flux is measured in the core plasma.

- Finally, HIBP becomes multipurpose diagnostics to study plasma electric potential and turbulence properties in the middle size toroidal devices.

\section{Acknowledgements}

The work of Kurchatov team (sections 2, 3.1 and 3.2) was funded by Russian Scientific Foundation, project 14-22-00193.

\section{References}

[1] Yu.N. Dnestrovskij et al., Development of heavy ion beam probe diagnostics, IEEE Trans. Plasma Sci. 22 (1994) 310.

[2] A.J.H. Donné, A.V. Melnikov and G. Van Oost. Diagnostics for radial electric field measurements in hot magnetized plasmas, Czech. J. Phys. 52 (2002) 1077.

[3] F.C. Jobes, R.L. Hickock, A direct measurement of plasma space potential, Nucl. Fusion 10 (1970) 195.

[4] D.R. Demers, et al., Radial electrostatic flux inferred from core measurements of potential and density fluctuations, Phys. Plasmas 8 (2001) 1278. 
[5] A.V. Melnikov et al., HIBP diagnostics on T-10, Rev. Sci. Instrum. 66 (1995) 317.

[6] I.S. Bondarenko et al., Installation of an advanced heavy ion beam diagnostic on the TJ-II stellarator, Rev. Sci. Instrum. 72 (2001) 583.

[7] A.V. Melnikov et al., Plasma potential evolution study by HIBP diagnostic during NBI experiments in the TJ-II stellarator, Fusion Sci. Technol. 51 (2007) 31.

[8] A.V. Melnikov et al., Plasma potential measurements by the heavy ion beam probe diagnostic in fusion plasmas: biasing experiments in the TJ-II stellarator and T-10 tokamak, Fusion Sci. Technol. 46 (2004) 299.

[9] A.V. Melnikov et al., Plasma potential and turbulence dynamics in toroidal devices (survey of T-10 and TJ-II experiments), Nucl. Fusion 51 (2011) 083043.

[10] E.J. Powers, Spectral techniques for experimental investigation of plasma diffusion due to polychromatic fluctuations, Nucl. Fusion 14 (1974) 74.

[11] A.V. Melnikov et al., The changes in plasma potential and turbulent particle flux in the core plasma measured by heavy ion beam probe during L-H transitions in the TJ-II stellarator, Nuclear Fusion 53 (2013) 092002.

[12] A.V. Melnikov et al., Alfv'en Eigenmodes Properties and Dynamics in the TJ-II Stellarator, Nucl. Fusion 52 (2012) 123004.

[13] L. Eliseev et al., Two point correlation technique for the measurements of poloidal plasma rotation by Heavy Ion Beam Probe, Plasma and Fusion Research Vol. 7, (2012) 2402064. 